\title{
The role of presenilins in Alzheimer's disease
}

\author{
Gopal Thinakaran
}

Department of Neurobiology, Pharmacology, and Physiology, The University of Chicago, Knapp R212, 924 East 57th Street, Chicago, Illinois 60637, USA.

Phone: (773) 834-3752; Fax: (773) 834-3808; Email: gopal@uchicago.edu.

In the past few years, 2 homologous polytopic membrane proteins termed presenilins (PSs) have been the focus of several research groups interested in the molecular mechanisms of Alzheimer's disease (AD). Mutations in the Presenilin 1 (PS1) and Presenilin 2 (PS2) genes account for the majority of the cases of familial earlyonset $\mathrm{AD}$ (see the Perspective by Tanzi in the previous issue of the JCI). Analysis of PS1 null mice and other model systems reveals a role for PS1 in development. Furthermore, biochemical and genetic evidence indicate that PS1 plays an important role in facilitating the proteolytic processing of certain proteins, including amyloid precursor protein (APP) and Notch1. Mutant PS proteins influence the $\gamma$-secretase-mediated processing of APP, cause a selective increase in the levels of highly fibrillogenic $A \beta 42$ species, and accelerate $A \beta$ deposition in the brains of transgenic mice. This apparent gain of function of mutant PS in AD seems to be distinct from the role of PS1 in development. Although PS1 interacts with several proteins, including members of the armadillo family of proteins, thus far none of the PS-interacting proteins have been shown to play a direct role in the enhanced production of $A \beta 42$ mediated by mutant PS. This review focuses on the unusual and fascinating aspects of PS metabolism, its role in facilitating the traf- ficking and cleavage of a set of membrane-bound proteins, and its role in the pathogenesis of AD.

The genetics and structure of the presenilins. Approximately $10 \%$ of all cases of Alzheimer's disease (AD) are estimated to be early-onset familial AD (FAD) and show autosomal dominant inheritance. Mutations in the Presenilin 1 (PS1) gene and the related gene Presenilin 2 (PS2), located on chromosomes 14 and 1, respectively, are causative in approximately $50 \%$ of pedigrees with FAD. Since 1995, more than 50 different mutations in PS1 have been described in over 80 families of various ethnic origins with early-onset FAD. With 2 notable exceptions (one, a deletion of 30 amino acids, and the other, insertion of 1 amino acid), mutations in presenilins are missense substitutions that result in single amino acid changes. In contrast to PS1 mutations, thus far only 2 mutations in PS2 have been described. These alleles occur in families with variable onset, autosomal dominant FAD. PS1 is a 467-amino acid polypeptide predicted to contain between 7 and 9 transmembrane-spanning domains and to include a hydrophilic "loop" region. A variety of biochemical and cell biologic approaches were used to determine the topology of PS1 and the Caenorhabditis elegans homologues SEL12 and HOP-1. These data generally predict that the $\mathrm{NH}_{2}$-terminus, the

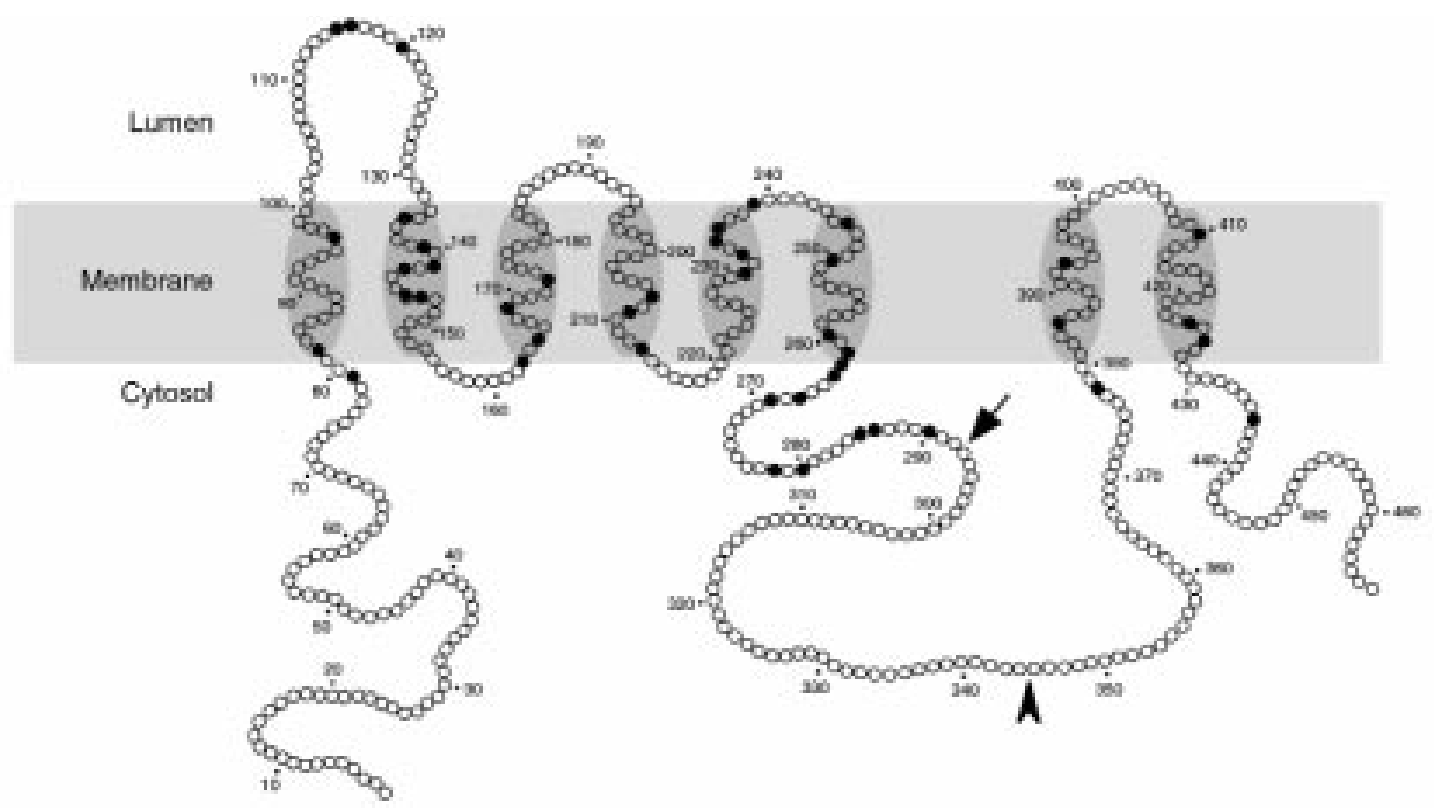

Figure 1

Schematic diagram of PS1 based on the 8 transmembrane domain model. A circle represents each amino acid of PS1, and residues mutated in FAD are depicted as closed circles. Predicted transmembrane domains are shaded. The arrow indicates the major site of regulated endoproteolysis (position 292-293); the arrowhead indicates the caspase cleavage site (position 344-345). 
$\mathrm{COOH}$-terminus, and the loop domain are exposed to the cytosol, as shown in Figure 1, where PS1 is depicted with 8 transmembrane domains. Alternate models with fewer transmembrane domains have also been presented wherein the $\mathrm{COOH}$-terminal hydrophobic domains are associated with, but do not span, the lipid bilayer.

Presenilin metabolism. Although PS1 is synthesized as a 42- to 43-kDa polypeptide, the preponderant PS1-related species that accumulate in vivo are 27 - to $28-\mathrm{kDa} \mathrm{NH}_{2}$ terminal (NTF) and 16 - to $17-\mathrm{kDa} \mathrm{COOH}$-terminal (CTF) proteolytic derivatives (1). Epitope mapping studies (1) and radiosequencing analysis (2) revealed that PS1 endoproteolysis occurs in the cytoplasmic loop domain, within a domain in which several of the identified FADlinked PS1 mutations occur. The $\mathrm{NH}_{2}$-terminus of the CTF is heterogeneous, with the 2 predominant species beginning at amino acids 293 and 299 (2). These findings are consistent with the demonstration that the FADlinked PS1 $\triangle E 9$ variant, which lacks exon 9 encoded sequences (amino acids 290-319), fails to be cleaved (1). It must be noted that lack of cleavage is not a general theme with all FAD-linked PS1 variants. Many FADlinked PS1 missense mutations seem not to affect endoproteolysis in cultured cells or transgenic mice or in the brains of patients with FAD (for example, see refs. 2-4). However, a few studies have provided evidence for incomplete processing of mutant PS polypeptides in transfected cells. One such mutation, C410Y, is inefficiently processed in a variety of cultured cells. Nevertheless, in brain samples from individuals with FAD, PS1 C410Y exists entirely as cleaved NTF and CTF, arguing against any processing defects in vivo. At present, neither the identity of the protease nor the physiological significance of PS1 proteolysis is known. Although autoproteolytic cleavage of PS1 has been proposed, there is no experimental evidence that PS1 or PS2, translated in vitro in the presence of microsomes, can generate NTF and CTF by autoproteolysis.

Analysis of human PS1 expression in brains of transgenic mice revealed a highly unusual aspect of the metabolism of presenilins. The levels of PS1 derivatives are remarkably disproportionate to levels of transgenederived mRNA or full-length human PS1. Intriguingly, in transfected cells overexpressing PS1 or PS2, a small fraction of newly synthesized full-length PS1 and PS2 is converted to stable fragments, whereas the majority of the protein fails to be processed and is rapidly degraded. These findings led to the proposal that the accumulation of human PS1-specific NTF and CTF is highly regulated and saturable. Attempts by several laboratories to establish a precursor-product relationship by conventional pulse chase analysis have not been fruitful. It is still unknown how only a subset of synthetic molecules is selected for the "cleavage" pathway and the remainder targeted for "rapid degradation." Remarkably, in mouse N2a cell lines and in brains of transgenic mice expressing human PS1, accumulation of human PS1 derivatives is accompanied by a compensatory and highly selective decrease in the steady-state levels of murine PS1 and PS2 derivatives. Similarly, the levels of murine PS1 derivatives are diminished in cultured cells overexpressing human PS2. Overexpression of the PS1 $\Delta E$ 9 variant, which fails to be cleaved, also resulted in compromised accumulation of murine PS1/PS2 derivatives; thus the "replacement" of murine PS1 and PS2 by overexpressed human PS1 takes place independent of endoproteolysis. These results are consistent with a model in which the abundance of PS1 and PS2 derivatives is coordinately regulated by competition for shared, but limiting, cellular factors (5).

It has been debated whether the full-length presenilins or the cleaved derivatives represent the "functional" molecule. In view of the demonstration of a paucity of fulllength PS1 and highly regulated accumulation of processed derivatives in vivo, it is highly likely that PS1 fragments are the "functional units." Moreover, human PS2 (6) and other presenilin homologues are also subject to endoproteolytic cleavage. These findings indicate that endoproteolysis of presenilins is a highly conserved process and, arguably, a processing event that regulates the accumulation of fragments. Nevertheless, it is important to note that the PS1 $\Delta \mathrm{E} 9$ variant is pathogenic, despite the lack of endoproteolytic cleavage. Thus, cleavage is not required for the pathogenic function of mutant presenilins in elevating the levels of $\mathrm{A} \beta 42$ and, perhaps, other biologic functions of presenilins as well. Recent studies provide evidence that the fragments derived from endoproteolysis and the noncleavable PS1 $\Delta$ E9 variant share several biochemical characteristics. For example, the sedimentation properties of PS1 $\triangle \mathrm{E} 9$ are similar to those of the cleaved PS1 NTF and CTF, a finding that offers an appealing notion that the uncleaved $\Delta \mathrm{E} 9$ variant retains the ability to associate with components of the "functional complex" (7), thus mimicking the normal properties of the wild-type PS fragments.

In our laboratory, we have used a variety of $\mathrm{COOH}$ terminally truncated and chimeric presenilin polypeptides to characterize the interaction between NTF and CTF. We observed that transgene-derived human PS1 NTF expressed in mouse N2a cells neither assembled with the endogenous CTF nor inhibited the cleavage of endogenous mouse PS1 and accumulation of resulting derivatives. Furthermore, in cells coexpressing PS1 and PS2, PS1- and PS2-derived fragments do not form mixed assemblies. In contrast, cells expressing a chimeric PS1/PS2 polypeptide formed PS1 NTF•PS2 CTF assemblies. These studies provide strong evidence that intramolecular associations between PS domains precede endoproteolytic processing (8). We speculate that a small fraction of newly synthesized presenilin polypeptides establish proper intramolecular associations during folding and proceed to interact with other components to form a high-molecular-weight complex. Future characterization of the high-molecular-weight complexes will facilitate further investigations into regulated endoproteolysis of presenilins and stable accumulation of derivatives.

FAD-linked PS1 mutations influence. APP metabolism The mechanisms by which FAD-linked PS1 and PS2 variants cause disease are not fully understood, but several important insights have emerged. The most provocative insight came from studies that examined the levels of different-length $A \beta$ peptides in conditioned medium from fibroblasts or in plasma from patients with FAD with mutations in PS1 or PS2. Proteolytic processing of 


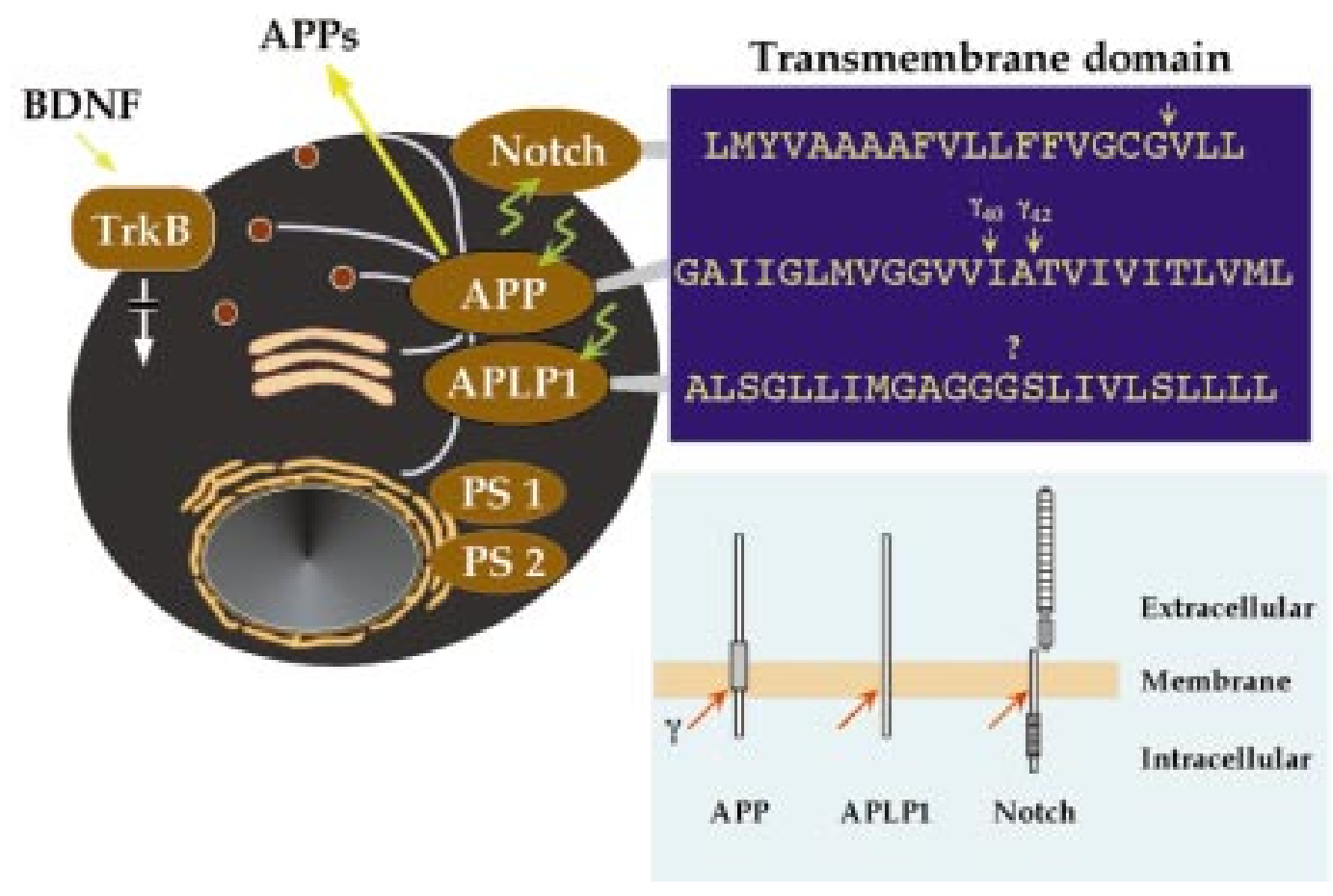

Figure 2

Effects of PS1 deficiency on membrane protein trafficking and cleavage. PS1-deficient neurons fail to secrete A $\beta$, the peptide derived from proteolytic processing of APP, owing to the lack of a so-called $\gamma$-secretase activity. Mutations in PS1 or PS2 enhance $\gamma 42$ cleavage. Despite the lack of sequence similarity near the $\gamma$-secretase cleavage site (see Transmembrane Domains box), APLP1 and Notch 1 are also cleaved inefficiently in PS1deficient cells. Interestingly, in neurons lacking PS1, the maturation and the ligand-dependent autophosphorylation activity of the receptor tyrosine kinase TrkB are also severely compromised. Thus, it is likely that PS1, which is predominantly localized in the endoplasmic reticulum, may influence trafficking and metabolism of selected membrane proteins, including APP, APLP1, Notch 1, and TrkB.

amyloid precursor protein (APP) releases into the extracellular space a set of 39-43 amino acid peptides collectively termed $A \beta$. Longer $A \beta$ peptides ( 42 or 43 amino acids in length) are more fibrillogenic than are shorter $\mathrm{A} \beta$ peptides (40 amino acids in length) and are more prevalent in the brain lesions of patients with $\mathrm{AD}$. Using highly sensitive sandwich ELISA assays with antibodies specific for the $\mathrm{COOH}$-terminus of $\mathrm{A} \beta 40$ and $A \beta 42$, Scheuner et al. demonstrated that FAD-linked PS1 and PS2 variants selectively enhance the proteolytic process that generates the $\mathrm{COOH}$-terminus of $\mathrm{A} \beta 42$ peptide (9). Subsequent studies documented the influence of mutant PS1 and PS2 on $A \beta 40$ and $A \beta 42$ production in transfected mammalian cells and in the brains of transgenic mice (for example, see refs. 3, 4, and 10). More recently, it was demonstrated that mutations in PS1 act synergistically with APP mutations to enhance production of $A \beta 42$ in transfected cells and accelerate brain amyloid deposition. Thus, mutations in presenilins increase the extracellular concentration of highly amyloidogenic $A \beta 42$ species, consequently fostering $A \beta$ deposition in the brain.

How mutant PS influences the production of $A \beta 42$ peptides remains uncertain, but these PS mutations appear to cause aberrant gain, rather than loss, of function. For example, the absence of nonsense or frameshift mutations that lead to truncated PS1 or PS2 argues against the loss-of-function model. Furthermore, an FAD-linked mutant PS1 allele was recently found to rescue the developmental abnormalities observed in PS1- deficient embryos. Moreover, expression of an artificial PS1 harboring 2 FAD-linked mutations exerted an even greater effect on $\mathrm{A} \beta 42$ peptide production than did PS1 variants harboring single mutations. The levels of $A \beta 42$ or $A \beta 40$ peptides in brains of mice with a single PS1 allele are no different from $A \beta$ levels in wild-type mice. Finally, $A \beta 42$ levels correlated with gene dosage in homozygous or heterozygous PS1 "knock in" mice carrying the I213T substitution. Thus, simple reduction in the level of wildtype PS1 is unlikely to account for the FAD-linked PS1 variant-mediated elevations in $A \beta 42$ levels.

One alternate proposal for the pathogenic function of FAD mutation is that mutant PS1 acts as a pathological "chaperone" to facilitate proteolytic cleavage by " $\gamma$-secretase" and selectively favors cleavage that generates $A \beta 42$. This idea was supported by earlier reports, which suggested that full-length PS1 and PS2 form stable heteromeric assemblies with APP in cultured mammalian cells $(11,12)$. Although attractive, this model is highly implausible, as the in vivo stoichiometry of PS1 and APP is approximately 1:100. Indeed, in a variety of experimental conditions that permit successful cross-linking or coprecipitation of PS NTF and CTF, or coisolation of APP and a bona fide interacting protein, Fe65, interactions between PS1 or PS2 and APP have not been detected $(13,14)$. Furthermore, higher-molecular-weight complexes containing PS derivatives do not cofractionate with APP on glycerol velocity gradients, arguing against the formation of stable APP•PS assemblies. It is conceivable that transient interactions occur between the trans- 
membrane domains of presenilins and APP, but the significance and, more importantly, the specificity of such an interaction at the membrane interface can only be established by well-controlled studies performed using models that closely resemble the in vivo situation. It must be noted that the conclusions regarding stable association between APP and PS were derived from transient transfection studies or analysis of stable cell lines overproducing both the proteins. Curiously, the 2 reports that inspired the idea reported that only the immature forms of APP associated with PS; neither study demonstrated association between PS1 and APP $\mathrm{CTF}$, the immediate precursor for $\mathrm{A} \beta 42(11,12)$.

Caspase-mediated cleavage of PS. In addition to the regulated endoproteolytic processing or presenilins just described, PS1 and PS2 also undergo additional cleavage dubbed "alternative cleavage," within the hydrophilic loop domain. First described by Kim et al. (15) in transfected H4 neuroglioma cell lines overexpressing PS2, alternative cleavages occur at consensus sites recognized by the caspase family of proteases, events that are effectively inhibited by the broad-spectrum caspase inhibitor, z-VAD-fmk. Full-length PS1, as well as PS1- or PS2derived CTFs, can be cleaved by caspases in transfected cells and cells induced to undergo apoptosis. Several members of the caspase family of proteases, including caspases 1, 3, 6, 7, 8, and 11, are capable of cleaving PS1 and PS2 in vitro. Interestingly, caspase-derived alternative PS1 NTF is prominent in cultured differentiating neurons. Kim et al. reported that cultured $\mathrm{H} 4$ neuroglioma cell lines expressing N141I mutant PS2 produced higher levels of the alternative CTF and suggested that these alternative cleavage fragments might play important roles in the pathogenesis of AD (15). In contrast to this report, no difference in the levels of NTF or CTF was detected in the brains of transgenic mice expressing wild-type or N141 mutant PS2 (16). Furthermore, in brains of transgenic mice expressing wild-type or a variety of mutant PS1 polypeptides, caspase-generated CTFs are not readily detectable (for example, see refs. 3, 4, and 17). Although these caspase-generated approximately 14-kDa PS1 CTFs were detected at low levels in brains of individuals harboring FAD-linked PS1 C410Y mutation, or sporadic AD cases, their abundance in $\mathrm{AD}$ brain samples was indistinguishable from that of controls (2). Four different FAD-linked PS1 variants also failed to show altered sensitivity to caspases in vitro. Two studies directly addressed the importance of caspase cleavage of presenilins on the elevated production of $A \beta 42$. In 1 study, it was demonstrated that inhibition of caspase cleavage by site-directed mutagenesis or by incubation with peptide aldehyde inhibitors did not diminish the pathogenic activity of mutant PS1 or PS2 on elevated $A \beta 42$ production. We recently observed that a large deletion within the cytoplasmic loop domain of PS1 or PS2, which includes the caspase cleavage site, had no effect on enhanced $A \beta 42$ production by mutant PS1 or PS2 polypeptides. Thus, it is highly unlikely that the caspase cleavage of PS1 and PS2 has a direct role in the elevated production of $A \beta 42$. However, it is unclear whether the alternative cleavage of presenilins plays any other role in the pathophysiology of AD.
A role for presenilin in apoptosis. The first clue that presenilins might regulate apoptosis emerged from a functional strategy designed to select genes involved in apoptosis. In this screen, a cDNA that codes for the COOH-terminal 103 residues of PS2 was found to rescue T-cell receptor-induced apoptosis in a mouse T-cell hybridoma (18). The protection offered by PS2 fragment was mediated by specific inhibition of Fas ligand induction and was not effective when cells were challenged with other apoptotic insults such as dexamethasone, actinomycin, or ceramide. Overexpression of fulllength PS2 reversed the protective effect, indicating that the COOH-terminal PS2 polypeptide acted as a competitive inhibitor of some interaction mediated by fulllength PS2 or by its normal COOH-terminal proteolytic product. Subsequent studies indicated that PS2 might also participate in neuronal apoptosis. Moreover, the expression of an antisense PS2 mRNA protected cells from apoptosis after treatment with glutamate or withdrawal of trophic factors, whereas expression of mutant PS1 or PS2 enhanced the sensitivity of cultured cells to a variety of proapoptotic insults (see ref. 19). Recent studies of hippocampal neurons from mice generated by "knock-in" of an FAD mutation also support this idea. Collectively, these studies suggest that the expression of mutant PS may influence neurodegeneration. One concern associated with several of the cell culture systems is the apparent induction of cell death initiated by the overexpression of wild-type PS1 and PS2 polypeptides in the absence of any apoptotic stimulus (for example, see ref. 15).

Direct in vivo evidence for a role for PS1 in neurodegeneration came from quantitative stereological analysis, which revealed a significant decrease in neuronal numbers in the frontal cortex and hippocampal CA1 fields of mice expressing human FAD-linked variants compared with mice expressing wild-type PS1 (20). Although there were no detectable extracellular A $\beta$ deposits in plaques, the mutant PS1 mice contained significantly more neurons with intracellular $A \beta 42$ deposits. It is not clear how the increase in $\mathrm{A} \beta 42$ production and increased apoptosis are related. Mutant presenilins are thought to regulate apoptosis via perturbed calcium homeostasis and enhanced oxyradical production and by influencing signaling pathways involving Akt/PKB and Jun kinase. Antioxidants, neurotrophic factors, and various experimental conditions that stabilize calcium homeostasis can suppress the deleterious properties of mutant presenilins. Nevertheless, the precise molecular mechanisms are not clearly defined. The recent finding that presenilins interact with $\mathrm{Bcl}-\mathrm{X}_{\mathrm{L}}$, a member of the antiapoptotic Bcl2 family of proteins, offers a potential mechanism by which presenilins might regulate apoptosis. However, it is unclear at present whether increased apoptotic response associated with FAD-linked mutations can be attributed to differential interaction between mutant presenilins and $\mathrm{Bcl}-\mathrm{X}_{\mathrm{L}}$.

Presenilin-interacting proteins. As already described here, PS-derived NTF and CTF are components of highmolecular-weight complexes. Several investigators recently used yeast 2-hybrid assays and candidate 
approaches to identify proteins that interact with various domains of PS1 or PS2. These efforts uncovered interactions between presenilins and a variety of wellknown and novel proteins including filamin, an actin binding protein; calcium-binding proteins termed calsenilin, calmyrin, and sorcin, mu-calpain; antiapoptotic molecule Bcl-X $\mathrm{X}_{\mathrm{L}}$ Rab11, a small GTPase involved in regulation of vesicular transport; brain G-protein, $G_{0}$; TPIP, a tetratricopeptide repeat containing protein; and glycogen synthase kinase- $3 \beta$, tau, Notch 1 and $\beta$-catenins. The C. elegans PS homologue SEL-12 was recently reported to interact with SEL-10, a Cdc4p-related protein. The most interesting information uncovered by the 2-hybrid binding studies is the interaction between PS1 and members of a family of armadillo-related proteins including $\beta$-catenin, $\gamma$-catenin, $\delta$-catenin, p0001, and neural-specific plakophilin. Although $\beta$-catenin is a multifunctional protein involved in Wnt signal transduction, cell adhesion, and tumor progression, the functional significance of PS/ $\beta$-catenin interaction is unclear. An earlier report suggested that wild-type PS1 stabilized $\beta$-catenin and that either the expression of FAD-linked mutant PS1 or the complete loss of PS1 resulted in increased degradation of $\beta$-catenin in cultured cells and in the brains of transgenic mice (21). In contrast, a recent study demonstrated that the half-life of $\beta$-catenin was markedly longer in $\mathrm{PS}^{-{ }^{--}}$cells and that overexpression of wild-type PS1 accelerated the turnover of $\beta$-catenin; mutant PS1 only partially influenced $\beta$ catenin turnover (22). Furthermore, approximately 35 to $90-\mathrm{kDa} \beta$-catenin polypeptides reported by Kang et al. (molecules that were thought to represent enhanced $\beta$-catenin degradation) were not detectable by pulsechase kinetic studies of $\beta$-catenin turnover by 2 laboratories $(22,23)$. The reason for the apparent discrepancies between these studies is not clear. In addition, there is disagreement on the issue whether PS2 interacts with $\beta$-catenin and catenin homologues. Nevertheless, FADlinked mutations in PS1 appear to cause defective intracellular trafficking of $\beta$-catenin (23). It is somewhat puzzling that neither the regulated metabolism of presenilins nor the enhanced production of $A \beta 42$ by FAD mutants appears to be influenced by any of the reported presenilin interacting proteins.

Presenilins facilitate intramembranous processing of APP, APLP1, and Notch 1. Analysis of APP processing in neurons from mice containing a targeted deletion of PS1 revealed that PS1 is required for $\gamma$-secretase cleavage of APP. Lack of PS1 leads to defects in secretion of A $\beta$ peptides and intracellular accumulation of APP CTFs bearing varying extents of the $A \beta$ region $(24,25)$. Although the biochemical mechanisms underlying this fascinating observation is not fully understood, 2 hypotheses have emerged: (a) PS1 is indeed the $\gamma$-secretase, or (b) PS1 plays an essential role in protein trafficking and compartmentalization of the $\gamma$-secretase and/or APP. Very recently Wolfe and colleagues presented evidence to support the suggestion that PS1 is itself a $\gamma$-secretase (26). They reported that mutating either of 2 aspartate residues in transmembrane domains 6 and 7 of PS1 leads to substantial reductions in $\mathrm{A} \beta$ secretion and accumulation of APP CTFs. Furthermore, microsomes pre- pared from cells expressing wild-type PS1 and not the aspartate mutant PS1 supported $\gamma$-secretase cleavage of APP CTF in an in vitro system. On the basis of these findings, the authors proposed that PS1 is either a cofactor for $\gamma$-secretase or is itself the $\gamma$-secretase. Interestingly, the aspartate mutant PS1 molecules are not subject to endoproteolysis, raising the possibility that PS1 endoproteolysis is autocatalytic and is mediated by an intrinsic aspartyl protease activity (26). However, as already discussed here, no available evidence indicates that authentic PS1 or PS2 NTF and CTF can be generated by autocatalysis. Thus, although the requirement of transmembrane aspartate residues in presenilins for APP processing is intriguing, confirmation that PS is the enzyme itself is lacking (see ref. 14) and will require reconstitution of the $\gamma$-secretase/presenilinase activities in artificial lipid bilayers using appropriate substrates and cellular factors.

It is remarkable that although PSs are required for the production of both $A \beta 40$ and $A \beta 42$, FAD-linked mutations in PS specifically influence the generation of $A \beta 42$ peptides. Analysis of a series of insertion and deletions within the transmembrane of APP indicates that the membrane positioning of APP can influence the length of $A \beta$ peptide generated by $\gamma$-secretase cleavage (27). On the basis of these studies, one could envision potential mechanisms by which mutant PS might selectively facilitate cleavage at $A \beta$ residue 42 by binding to APP CTFs. While there is sufficient evidence indicating that $\gamma$-secretase interacts with APP CTF and that mutations in the transmembrane domain of APP interfere with this interaction, no interaction between PS and APP CTF has been found (see ref. 14). Recent biochemical and immunocytochemical studies argue strongly that $A \beta 40$ and $A \beta 42$ are generated at distinct subcellular compartments. Moreover, pharmacological evidence supports the existence of multiple proteolytic activities that release $\mathrm{A} \beta 40$ or $\mathrm{A} \beta 42$. Thus, the connection between FAD-linked presenilin mutations, its influence on the $\gamma$-secretase, and enhanced production of $A \beta 42$ is far from clear.

Regardless of the mechanism by which PS facilitates APP cleavage, it is now also apparent that loss of PS1 activity also interferes with intramembranous processing of Notch 1 (Figure 2) in Drosophila and mammalian cells (28-30). As De Strooper et al. reported (30), mNotch $\Delta \mathrm{E}$, a constitutively active Notch 1 polypeptide, is inefficiently processed at an intramembranous site in PS1-deficient immortalized fibroblasts. This cleavage event is critical for Notch function because it releases the intracellular domain from the membrane; upon release, the intracellular domain is translocated to the nucleus, where it activates transcription of Notch target genes. Notch undergoes at least 2 additional cleavages in its extracellular domain, mediated by the furin and Kuzbanian proteases. The constitutively active mNotch $\Delta \mathrm{E}$ construct used by De Strooper et al. (30) lacks most of the extracellular domain of Notch, including the EGF and Notch/Lin-12 repeats. It will be of considerable interest to evaluate the role of PS1 in proteolysis of full-length forms of Notch and influences on ligand-mediated Notch proteolysis/signaling. In this regard, Struhl and Greenwald have demonstrat- 
ed that Drosophila with null mutations in the presenilin gene exhibit extensive neuronal hyperplasia and have wing phenotypes similar to those observed in flies lacking Notch. In the absence of PS, ligand-dependent Notch signal transduction is abolished. Although nuclear entry of a constitutively active cytosolic form of Notch is unimpaired in these flies, the cytosolic domain derived from transmembrane forms of Notch fail to enter the nucleus (29). Ye et al. have also described neurogenic phenotypes in Drosophila with loss-of-function alleles of presenilin. However, in contrast to the studies of Struhl and Greenwald, Ye et al. find that neither membrane-tethered nor cytosolic forms of Notch 1 show differences in signaling or neuronal specification in Psn-deficient wing discs (28). These studies argue against a direct mechanism of action of PS on proteolytic processing of Notch 1 to release the nuclear-translocated cytoplasmic domain. Instead, they suggest that PS acts upstream of Notch signal transduction by influencing ligand binding or the assembly of the furin-cleaved $\mathrm{NH}_{2}$ - and $\mathrm{COOH}$-terminal Notch fragments into functional heterodimers that are substrates for subsequent extracellular cleavage by Kuzbanian or other proteases.

Although there are obvious parallels between the proteolysis of APP and Notch 1, it is highly unlikely that PS1 is the endoprotease involved in intramembranous cleavage of each substrate. Little, if any, homology exists between the Notch 1 and APP transmembrane domains, and the positions of each scissile bond with respect to the boundaries of each transmembrane helix are quite different (Figure 2). A more attractive hypothesis holds that PS1 influences endoproteolysis of APP and Notch 1 indirectly, by altering trafficking and cocompartmentalization of each substrate and the elusive protease(s). Indeed, it was recently demonstrated that CTF derived from the APP homologue, APLP1, also accumulates in $\mathrm{PS}^{-/-}$neurons (25). Because the APP and APLP1 transmembrane domains have very limited homology, it is inconceivable that PS1 plays a role as a specific $\gamma$-secretase involved in the cleavage of both substrates. Rather, a broader role for PS1 in directing membrane-bound CTFs derived from APP family members or other transmembrane proteins to appropriate cleavage and/or degradation compartments might be envisioned. In this regard, the loss of PS1 expression also affects the biology of other integral membrane glycoproteins, such as the receptor tyrosine kinase, TrkB. The rate of maturation and brain-derived neurotrophic factor-mediated autophosphorylation of TrkB is severely compromised in $P S 1^{-/-}$neurons (25). In support of these observations, Levitan and Greenwald have demonstrated that in C. elegans, reduced SEL-12 activity results in diminished apical membrane accumulation and signaling of LIN-12/Notch in vulval precursor cells (31). Given that SEL-12 was localized primarily to the $\mathrm{ER} /$ Golgi, this finding is consistent with a role of the molecule in membrane protein trafficking.

\section{Conclusions}

Intramembranous cleavage by 1 or more enzymes termed " $\gamma$-secretases" is the last step in the metabolism of APP that generates 39-43 amino acid peptides termed
$A \beta$. $A \beta 40$ and $A \beta 42$ are the predominant soluble $A \beta$ peptides in the brain, and $A \beta 42$ is the most amyloidogenic of the $A \beta$ species that are deposited in senile plaques. Inhibition of $A \beta$ production by interfering with $\gamma$-secretases has been considered as one of the potential therapeutic targets for the treatment of Alzheimer's disease (see the Perspective by Wagner and Munoz in this issue of the JCI). The recent findings that APP and Notch (and conceivably other unidentified transmembrane proteins) might share proteolytic processing pathways compel us to reconsider the merit of this strategy. Nevertheless, the evidence that presenilins are required for proteolytic processing/trafficking of a set of transmembrane proteins, including APP and homologues, Notch 1, and TrkB, raises the compelling question of how these processes are influenced by FAD-linked mutations. Future development of inhibitors that specifically target APP or Notch cleavage will further our understanding of the precise role PS plays in $\gamma$-secretase cleavage of APP and other substrates. Much more work is needed to understand the normal biologic functions of presenilins in the adult brain and the effects of pathogenic mutations on these processes during aging. For example, there is little information available regarding the influence of mutant presenilins on synaptic structure and function $(32,33)$. For a thorough understanding of the biologic functions of presenilins, it will be necessary to generate conditional and tissue-specific "knock out" mouse models and to identify the components of the functional presenilin complexes.

\section{Acknowledgments}

The author thanks Sangram S. Sisodia (University of Chicago) and David R. Borchelt (Johns Hopkins University School of Medicine) for insightful comments and discussion. The author is supported by grants from the National Institutes of Health (1PO1 AG14248) and from the Alzheimer's Association and the Adler Foundation. Due to space limitations, it was not possible to cite several important primary reports that are discussed here.

1. Thinakaran, G., et al. 1996. Endoproteolysis of presenilin 1 and accumulation of processed derivatives in vivo. Neuron. 17:181-190.

2. Podlisny, M.B., et al. 1997. Presenilin proteins undergo heterogeneous endoproteolysis between Thr291 and Ala299 and occur as stable N-and C-terminal fragments in normal and Alzheimer brain tissue. Neurobiol. Dis. 3:325-337.

3. Borchelt, D.R., et al. 1996. Familial Alzheimer's disease-linked presenilin 1 variants elevate Abeta1-42/1-40 ratio in vitro and in vivo. Neuron. 17:1005-1013.

4. Duff, K., et al. 1996. Increased amyloid-beta42(43) in brains of mice expressing mutant presenilin 1. Nature. 383:710-713.

5. Thinakaran, G., et al. 1997. Evidence that levels of presenilins (PS1 and PS2) are coordinately regulated by competition for limiting cellular factors. J. Biol. Chem. 272:28415-28422.

6. Kim, T.W., et al. 1997. Endoproteolytic cleavage and proteasomal degradation of presenilin 2 in transfected cells. J. Biol. Chem. 272:11006-11010.

7. Capell, A., et al. 1998. The proteolytic fragments of the Alzheimer's disease-associated presenilin-1 form heterodimers and occur as a 100-150kDa molecular mass complex. J. Biol. Chem. 273:3205-3211.

8. Saura, C.A., et al. 1999. Evidence that intramolecular associations between presenilin domains are obligatory for endoproteolytic processing. J. Biol. Chem. 274:13818-13823.

9. Scheuner, D., et al. 1996. Secreted amyloid beta-protein similar to that in the senile plaques of Alzheimer's disease is increased in vivo by the presenilin 1 and 2 and APP mutations linked to familial Alzheimer's disease. Nat. Med. 2:864-870.

10. Tomita, T., et al. 1997. The presenilin 2 mutation (N141I) linked to familial Alzheimer disease (Volga German families) increases the secre- 
tion of amyloid beta protein ending at the $42 \mathrm{nd}$ (or 43rd) residue. Proc. Natl. Acad. Sci. USA. 94:2025-2030.

11. Weidemann, A., et al. 1997. Formation of stable complexes between two Alzheimer's disease gene products: presenilin-2 and beta-amyloid precursor protein. Nat. Med. 3:328-332.

12. Xia, W., Zhang, J., Perez, R., Koo, E.H., and Selkoe, D.J. 1997. Interaction between amyloid precursor protein and presenilins in mammalian cells: implications for the pathogenesis of Alzheimer disease. Proc. Natl. Acad. Sci. USA. 94:8208-8213.

13. Thinakaran, G., et al. 1998. Stable association of presenilin derivatives and absence of presenilin interactions with APP. Neurobiol. Dis. 4:438-453.

14. Haass, C., and Mandelkow, E. 1999. Proteolysis by presenilins and the renaissance of tau. Trends Cell Biol. 9:241-244.

15. Kim, T.W., Pettingell, W.H., Jung, Y.K., Kovacs, D.M., and Tanzi, R.E. 1997. Alternative cleavage of Alzheimer-associated presenilins during apoptosis by a caspase-3 family protease. Science. 277:373-376.

16. Oyama, F., et al. 1998. Mutant presenilin 2 transgenic mouse: effect on an age-dependent increase of amyloid beta-protein 42 in the brain. J. Neurochem. 71:313-322.

17. Citron, M., et al. 1997. Mutant presenilins of Alzheimer's disease increase production of 42- residue amyloid beta-protein in both transfected cells and transgenic mice. Nat. Med. 3:67-72.

18. Vito, P., Lacana, E., and D'Adamio, L. 1996. Interfering with apoptosis: $\mathrm{Ca}(2+)$-binding protein ALG-2 and Alzheimer's disease gene ALG-3. Science. 271:521-525.

19. Mattson, M.P., Guo, Q., Furukawa, K., and Pedersen, W.A. 1998. Presenilins, the endoplasmic reticulum, and neuronal apoptosis in Alzheimer's disease. J. Neurochem. 70:1-14.

20. Chui, D.H., et al. 1998. Both N-terminal and C-terminal fragments of presenilin 1 colocalize with neurofibrillary tangles in neurons and dystrophic neurites of senile plaques in Alzheimer's disease. J. Neurosci. Res. 53:99-106.

21. Zhang, Z., et al. 1998. Destabilization of beta-catenin by mutations in presenilin-1 potentiates neuronal apoptosis. Nature. 395:698-702.
22. Kang, D.E., et al. 1999. Presenilin 1 facilitates the constitutive turnover of beta-catenin: differential activity of Alzheimer's disease-linked PS1 mutants in the beta-catenin-signaling pathway. J. Neurosci. 19:4229-4237.

23. Nishimura, M., et al. 1999. Presenilin mutations associated with Alzheimer disease cause defective intracellular trafficking of beta-catenin, a component of the presenilin protein complex. Nat. Med. 5:164-169.

24. De Strooper, B., et al. 1998. Deficiency of presenilin-1 inhibits the normal cleavage of amyloid precursor protein. Nature. 391:387-390.

25. Naruse, S., et al. 1998. Effects of PS1 deficiency on membrane protein trafficking in neurons. Neuron. 21:1213-1221.

26. Wolfe, M.S., et al. 1999. Two transmembrane aspartates in presenilin-1 required for presenilin endoproteolysis and gamma-secretase activity. Nature. 398:513-517.

27. Murphy, M.P., et al. 1999. Gamma-secretase, evidence for multiple proteolytic activities and influence of membrane positioning of substrate on generation of amyloid beta peptides of varying length. J. Biol. Chem. 274:11914-11923.

28. Ye, Y., Lukinova, N., and Fortini, M.E. 1999. Neurogenic phenotypes and altered Notch processing in Drosophila presenilin mutants. Nature. 398:525-529.

29. Struhl, G., and Greenwald, I. 1999. Presenilin is required for activity and nuclear access of Notch in Drosophila. Nature. 398:522-525.

30. De Strooper, B., et al. 1999. A presenilin-1-dependent gamma-secretase-like protease mediates release of Notch intracellular domain. Nature. 398:518-522.

31. Levitan, D., and Greenwald, I. 1998. Effects of SEL-12 presenilin on LIN12 localization and function in Caenorhabditis elegans. Development. 125:3599-3606.

32. Wong, T.P., Debeir, T., Duff, K., and Cuello, A.C. 1999. Reorganization of cholinergic terminals in the cerebral cortex and hippocampus in transgenic mice carrying mutated presenilin-1 and amyloid precursor protein transgenes. J. Neurosci. 19:2706-2716.

33. Parent, A., Linden, D.J., Sisodia, S.S., and Borchelt, D.R. 1999. Synaptic transmission and hippocampal long-term potentiation in transgenic mice expressing FAD-linked presenilin 1. Neurobiol. Dis. 6:56-62. 